\title{
Alpha1,2-fucosyl transferase gene, the key enzyme of Lewis y synthesis, promotes Taxol resistance of ovarian carcinoma through apoptosis-related proteins
}

\author{
F.-F. LI ${ }^{1, *}$, D. SHA ${ }^{2, *}$, X.-Y. QIN ${ }^{1}$, C.-Z. LI ${ }^{1, *}$, B. LIN $^{3}$ \\ ${ }^{1}$ Department of Obstetrics and Gynecology, Shandong Provincial Hospital affiliated to Shandong Universtiy, Jingwu Road, Huaiyin District, \\ Jinan, 250021, China; ${ }^{2}$ Department of medical oncology, Shandong Provincial Hospital affiliated to Shandong Universtiy, Jingwu Road, Huaiyin \\ District, Jinan, 250021, China; ${ }^{3}$ Department of Obstetrics and Gynecology, Shengjing Hospital of China Medical University, 36 Sanhao Street, \\ Heping District, Shenyang, 110004, China
}

${ }^{*}$ Correspondence: $15168860882 @ 163 . c o m$

"Contributed equally to this work.

Received August 23, 2017 / Accepted October 18, 2017

\begin{abstract}
We aimed to investigate the role of FUT1 gene in Taxol resistance and to explore its mechanism in epithelial ovarian cancer. Three ovarian cancer cell lines, ES-2, SK-OV-3 and OVCAR-3 were selected from epithelial ovarian cancer in this experiment. Western blot was used to validate the protein expression level of FUT1 and the apoptosis proteins. The expression level of the corresponding carrier was validated by RT-PCR. Transfection and isolation of stable transfectants were carried out to establish the cell line models. The different concentrations of Taxol on the inhibition of cell growth rate was measured by MTT, in which Taxol resistance profiling in ovarian cancer cells was determined by IC50 data. Flow cytometry was conducted to compare cell apoptosis ability. Caspase-3 activity and the apoptosis proteins were measured by colorimetry and western blot, respectively, to further compare the cell apoptosis ability in different groups. To demonstrate the inhibition of miR-FUT1 combined with Taxol therapy against ovarian cancer, xenograft assay was carried out for the in vivo effect. The western blot results indicate that FUT1 is expressed in all of the ovarian cancer cells with different expression level: ES-2 > SK-OV-3 > OVCAR-3. Besides, FUT1 siRNA was used in the maximum expression of FUT1 cell line ES-2, or over-expression plasmid was used in the minimum expression of FUT1 cell line OVCAR-3, to establish stable expression cell lines. After the treatment with Taxol, the inhibition rate of Taxol was obviously decreased with the established cell model above, and the IC50 level was significantly increased in the FUT1 over-expression + Taxol group (p<0.05, respectively). Additionally, after the treatment with Taxol, the results of flow cytometry demonstrated that the apoptosis rate was increased or decreased obviously in the FUT1 low or high expression group compared with the control group ( $\mathrm{p}<0.05$, respectively). Furthermore, the results of western blot indicated that the expression of the anti-apoptotic proteins such as survivin, XIAP, BCL-2 and BCL-xL was significantly decreased or increased in the FUT1 low or high expression group compared with the control group ( $\mathrm{p}<0.05$, respectively), and the determination of pro-apoptotic protein caspase- 3 enzyme activity test demonstrated that the caspase- 3 enzyme activity was increased or decreased obviously in the FUT1 low or high expression group compared with the control group ( $\mathrm{p}<0.05$, respectively). Finally, we verified the inhibition effect of siRNA-FUT1 combined with Taxol therapy against ovarian cancer through xenograft assay in vivo. Our results suggest that FUT1 combined with Taxol could promote Taxol resistance and inhibit cell apoptosis by raising the expression of anti-apoptotic proteins such as survivin, XIAP, BCL-2, and BCL and inhibiting caspase-3 enzyme activity. Thus, FUT1 might serve as a therapeutic target for ovarian cancer.
\end{abstract}

Key words: FUT1, Lewis y, Taxol resistance, ovarian cancer, apoptosis

Ovarian cancer is a malignancy with the fifth mortality in female malignant tumors and leads to the highest mortality rate in gynecological cancers, of which epithelial ovarian carcinoma accounts for $85-90 \%$ as the most common pathologic type. It is estimated that there will be 22,440 Americans diagnosed with ovarian cancer in 2017, and 14,080 of them will die from the disease [1]. Surgery with chemotherapy is still the main treatment for ovarian cancer. However, the high mortality rate of ovarian cancer is associated with the difficulties of early detection, and the majority experience relapses 
within two years $[2,3]$. Chemoresistance after chemotherapy makes the therapy very challengeable, and has been one of the main reasons for the high mortality of ovarian cancer [4]. Therefore, it is urgent to discover new treatment strategies for reducing the chemoresistance occurrence to improve prognosis.

Tumor related Lewis y antigen is a difucosylated oligosaccharide containing two fucoses, which is carried by glycoproteins and glycolipids on cell surface, and it has been widely used in early screening and biological treatment [5-8]. Over-expression of Lewis y antigen is usually found in human cancers (including breast, ovary, prostate, and colon cancers), especially in $75 \%$ of ovarian tumors and is associated with tumors' pathological staging and poor prognosis [5-8]. The studies have reported that Lewis y could stimulate the IGF-1R expression to promote the occurrence and development of ovarian cancers, regulate the epidermal growth factor receptor pathway to stimulate the growth of ovarian cancer cells, and promote the proliferation of ovarian cancer cells through the PI3K/Akt signaling pathway [5-8]. In our previous studies, we found that Lewis $y$ antigen is involved in ovarian cancer Taxol resistance, but the possible mechanism still remains unknown. Alpha 1,2-fucosyl transferase (FUT1) gene, which catalyzes the fucosylation of Lewis y, plays an important role in the above mechanism.

In the present study, we evaluated the FUT1 expression in three kinds of ovarian cancer cell lines and established the stable FUT1 expression cell lines for comparison. Then we demonstrated that FUT1 combined with Taxol could promote Taxol resistance and inhibit cell apoptosis. Finally, we explored the preliminary mechanism: FUT1 could promote Taxol resistance by increasing the expression of anti-apoptotic proteins such as survivin, XIAP, BCL-2, and the BCL and inhibiting the caspase- 3 enzyme activity. Thus, FUT1 might serve as a therapeutic target for ovarian cancer.

\section{Materials and methods}

Cell culture and treatment. Ovarian cancer cells ES-2, OVCAR-3, and SK-OV-3 (obtained from Saier Biotechnology INC, Tianjin, China) were cultured in RPMI 1640 supplemented with $10 \% \mathrm{FBS}$ (GIBCO BRL, USA) at $37^{\circ} \mathrm{C}$ in $5 \% \mathrm{CO}_{2}$ atmosphere. Stable transfectants were maintained in complete DMEM supplemented with $0.5 \mathrm{mg} / \mathrm{mL}$ G418. Cells were routinely checked for Mycoplasma contamination (Stratagene, La Jolla, CA, USA). For western blot assays, sub-confluent cell layers were rendered quiescent by serum starvation for 12-24 h. Cells were subsequently stimulated by addition of medium containing or lacking paclitaxel $(10 \mathrm{~mL} / 60 \mathrm{mg}, \mathrm{H} 20083956$ approved by the state, Hainan Sinochem conjointe Pharmaceutical Industry Co., Ltd, China) with different concentrations $(0 \mu \mathrm{M}, 1 \mu \mathrm{M}, 5 \mu \mathrm{M}$, and $20 \mu \mathrm{M})$. Four cell groups were involved in the experiment: 1) interference group (ES-2-shR-FUT1), 2) interference with the control group (ES-2-pSilencer), 3) over-expression of the experimental group (OVCAR-3-FUT1), 4) over-expression of the control group (OVCAR-3-pcDNA3.1).

Western blot analysis. For western blotting, cells were rinsed with PBS and $1 \mathrm{~mL}$ of RIPA lysis buffer was added. The supernatants were collected and stored at $-80^{\circ} \mathrm{C}$ after centrifugation. Protein content was measured by the protein assay BCA kit (Beyotime Biotechnology, China) and equal amounts of protein were loaded on SDS-PAGE gels. Subsequently, proteins were transferred to PVDF membranes (Millipore, USA), and the condition was $100 \mathrm{~mA}$ for $90 \mathrm{~min}$, before being detected with anti-FUT1 antibody (Abcam, England, 1:1,000); HRP-rabbit anti-IgG (Sigma Chemical; 1:10,000). Immunoreactive bands were visualized by LabWorksTM gel imaging and analysis system (UVP, USA), enhanced Chemiluminescence Substrate was used as secondary antibodies.

Transfection and isolation of stable transfectants. Lipofectamine2000 Reagent (Invitrogen, USA), Type B plasmid small rapid extraction kit (BioDev-Tech. Co.,Ltd, Beijing); XL1-Blue (Stratagene, USA); Type restriction endonucleases BamHI, EcoRI (Takara, Japan); pcDNA3.1(+)/ FUT1 plasmids were established by Self biotechnology (Tianjin), and the primers were as follow: FUT1-S-BamHI: 5'CGCGGATCCGCCACCATGTGGCTCCGGAGCCATCGT3'; FUT1-AS-EcoRI: 5'CCGGAATTCAGGCTTAGCCAATGT; pSilencer2.1-U6/shR-FUT1 plasmids establishment and the primers:

FUT1-shR1-top 5'-GATCCGCCGGTTTGGTAATCAGATGGCTCGAGCCATCTGATTACCAAACCGGCTTTTTGA-3', FUT1-shR1-Bot 5'-AGCTTCAAAAAGCCGGTTTGGTAATCAGATGGCTCGAGCCATCTGATTACCAAACCGGCG-3', FUT1-shR2-top 5'-GATCCGCGGACTTGAGAGATCCTTTCCTCGAGGAAAGGATCTCTCAAGTCCGCTTTTTGA-3', FUT1-shR2-Bot 5'-AGCTTCAAAAAGCGGACTTGAGAGATCCTTTCCTCGAGGAAAGGATCTCTCAAGTCCGCG-3', FUT1-shR3-top 5'-GATCCGGGACTATCTGCAGGTTATGCCTCGAGGCATAACCTGCAGATAGTCCCTTTTTGA-3', FUT1-shR3-Bot 5'-AGCTTCAAAAAGGGACTATCTGCAGGTTATGCCTCGAGGCATAACCTGCAGATAGTCCCG-3'.

A fragment of the rat FUT1 FTA complementary DNA fragment (368 bp, GENB/EMBL accession number L26009) was inserted at the unique EcoRI site in the anti-sense orientation as determined by sequencing. The final concentration of siRNA control and FUT1 was $100 \mathrm{nM}$. PROb cells grown in $60 \mathrm{~mm}$ Petri dishes were then transfected with $10 \mu \mathrm{g}$ of recombinant plasmid using lipofectamine according to the instructions (GIBCO, Paisley, UK). Fresh medium was added and replaced $24 \mathrm{~h}$ and $48 \mathrm{~h}$ later, respectively, by selective medium containing $0.2 \mathrm{mg} / \mathrm{mL}$ G418 (GIBCO). Individual colonies were picked, cloned and expanded.

MTT assay. The cells were seeded in a 96-well plate at $1 \times 10^{4}$ cells/well (three replicates), incubated at $37^{\circ} \mathrm{C}$ for $24 \mathrm{~h}$, and then cultured in DMEM (with 10\% FCS) containing paclitaxel. Cells were cultured in $5 \% \mathrm{CO}_{2}$ at $37^{\circ} \mathrm{C}$ for $24 \mathrm{~h}$, and then $10 \mu \mathrm{L}$ of $5 \mathrm{mg} / \mathrm{mL}$ MTT was added. After $4 \mathrm{~h}$ of subsequent culture, $100 \mu \mathrm{L}$ of DMSO was added to terminate 
the reaction, and the absorbance value was detected at 490 $\mathrm{nm}$ using a microplate reader (Elx808; Bio-Tek Instruments, Vermont, USA).

Cell inhibition rate $=1-($ treatment group OD - blank hole OD)/(blank control group OD - hole OD) $\times 100 \%$.

IC50 calculation method: using GraphPad Prism 5 software calculation.

Flow cytometry. Cultured cells were trypsinized and counted. A total of $5 \times 10^{5}$ viable cells were incubated for $30 \mathrm{~min}$ at $4^{\circ} \mathrm{C}$ with antibodies diluted in phosphate-buffered saline (PBS) containing $1 \%$ bovine serum albumin (BSA). Cells were rinsed in PBS-BSA $1 \%$ and incubated with the secondary antibody Alexa Fluor 488 (Invitrogen) for $30 \mathrm{~min}$ at $4{ }^{\circ} \mathrm{C}$. After washing, fluorescent analysis was carried out using a FACScan (Becton Dickinson, Franklin Lakes, NJ, USA).

Caspase- 3 activity was measured by colorimetry. The supernatant of cells treated with or without paclitaxel was removed and washed with PBS. Pyrolysis liquid was added and set on ice. After centrifugation for $5 \mathrm{~min}$ at $1,500 \mathrm{r} / \mathrm{min}$ $(\mathrm{r}=15 \mathrm{~cm})$, DEVP-PNA was then added to the supernatant. After incubation at $37^{\circ} \mathrm{C}$ for $1 \mathrm{~h}$, the absorbance at $405 \mathrm{~nm}$ wavelength was measured by microplate reader. Standard curve was plotted at $405 \mathrm{~nm}$ with standard PNA solutions at various concentrations. Caspase- 3 activity units (U) were calculated using the following equation. The experiment was repeated four times.

$\mathrm{U}(\%)=\mathrm{A} 405$ (Experimental group)/A405(Control group) $\times 100 \%$.

Xenograft assays in vivo. The animal study was carried out in accordance with the guidelines approved by the Animal Experimentation Ethics Committee of affiliated hospital of Shandong Provincial Hospital affiliated to Shandong University. The protocol was approved by the committee, all surgeries were performed under sodium pentobarbital anesthesia, and all efforts were made to minimize the suffering. Athymic $\mathrm{Balb} / \mathrm{c}$ nude mice (aged 6 weeks) were provided by Slac Laboratory Animal Co. Ltd. (Shanghai, China), and the mice were housed in a pathogen-free animal facility and randomly assigned to the control or experimental group (four mice per group). $2 \times 10^{6}$ related cells were suspended in $0.1 \mathrm{~mL}$ of serum-free RPMI 1640 before injecting into the right subaxillary. And the mice were divided into 4 groups on day 14 (the tumor volume reached about $50-100 \mathrm{~mm}^{3}$ ): control group (pSilence2.1), siR-FUT1, pSilence2.1+Taxol and siR-FUT1+Taxol group. The mice were injected with siRNA control (transfected with vector), siR-FUT1, siRNA control+Taxol, siR-FUT1+Taxol. The final concentration of the intratumorally-injected siRNA control or siR-FUT1 was $100 \mathrm{nM}, 0.01 \mathrm{~mol}$ each time. Further, Taxol was intraperitoneally injected at $10 \mathrm{mg} / \mathrm{kg}$ into the mice. The tumors were measured by vernier caliper, and the following formula was used to calculate the tumor volume: tumor volume $\left(\mathrm{mm}^{3}\right)$ $=$ tumor length $(\mathrm{mm}) \times$ tumor width $(\mathrm{mm}) \times$ tumor width $(\mathrm{mm}) / 2$. Mice were killed 21 days upon inoculation, and the final volume of tumor tissues was determined.
Statistical analysis. The SPSS 12.0 statistical analysis software was used, while the analysis of variance was employed. A $p<0.05$ was regarded as statistically significant.

\section{Results}

The protein expression of FUT1 in ovarian cancer cell lines, and the establishment of stable cell lines with interference-low and over-expression-high expression of FUT1. To prove the FUT1 protein expression in ovarian cancer cells, we employed three types of epithelial ovarian cancer cell lines: ES-2, SK-OV-3 and OVCAR-3. The western blot results indicate that FUT1 is expressed in all the above ovarian cancer cells, and the expression level is different: ES-2 > SK-OV-3 > OVCAR-3 (Figure 1A). To further compare the FUT1 protein expression in different ovarian cancer cell lines, we used FUT1 siRNA (Figure 1B) and/or over-expression plasmid (Figure 1C) to establish stable expression cell lines. And then we used FUT1 siRNA (shR1, 2 and 3 were used and we chose shR3 owing to the most effective result: the protein level of FUT1 was significantly lower in the shR2/3-FUT1 group than that in the control group NC, $\mathrm{p}<0.05$, respectively, Figure $1 \mathrm{~B}$ ) for the maximum expression of FUT1 cell line ES-2, and/or overexpression plasmid pcDNA3.1-FUT1 (the protein level of FUT1 was significantly higher in the FUT1 group than that in the control group pcDNA3.1(+), $\mathrm{p}<0.05$, Figure1C) for the minimum expression of FUT1 cell line OVCAR-3, to establish the stable expression cell lines to facilitate comparison, respectively. The results of the western blot for the different groups of the above established cell lines indicated that the protein level of FUT1 was significantly lower in the shR-FUT1 group than that in the control group pSilence2.1 and/or the protein level of FUT1 was significantly higher in the group than that in the control group pcDNA3.1 (+) $(p<0.05$, respectively, Figure 1D). To sum up, we established cell model lines of the interference-low and the over-expression-high expression group for further research.

The loss or the over-expression of FUT1 inhibited or promoted Taxol resistance. By using the established cell model above, after the treatment with the Taxol, the expression level of FUT1 was significantly decreased compared with the control group pSilence 2.1 transfected with the control shR $(p<0.05$, Figure 1D), the inhibition rate of Taxol was obviously increased and the IC50 level was significantly decreased in the shR3-FUT1 group ( $\mathrm{p}<0.05$, respectively, Figure $2 \mathrm{~A})$. Besides, compared with the control group pcDNA3.1(+) transfected with the empty plasmid, the expression level of FUT1 was significantly increased $(p<0.05$, Figure $1 D)$, the inhibition rate of Taxol was obviously decreased and the IC50 level was significantly increased in the FUT1 group ( $\mathrm{p}<0.05$, respectively, Figure $2 \mathrm{~A}$ ). The results demonstrate that the loss or the over-expression of FUT1 combined with Taxol inhibited or promoted Taxol resistance. In a word, the results proved that FUT1 could promote Taxol resistance. 
A

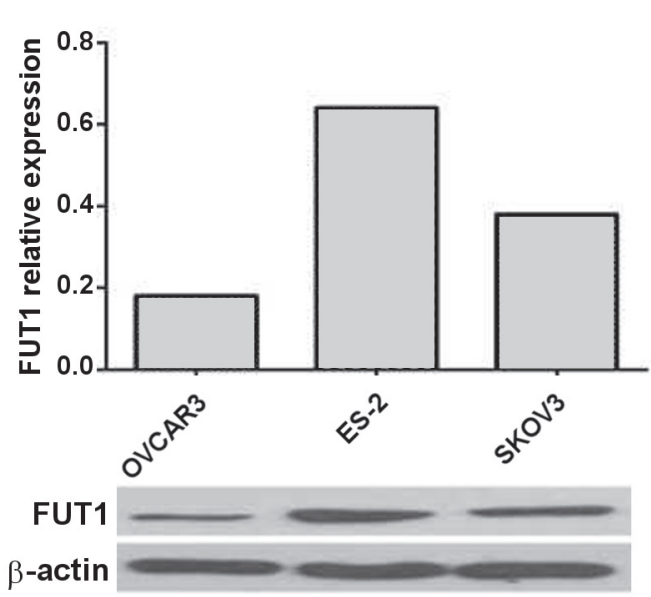

C
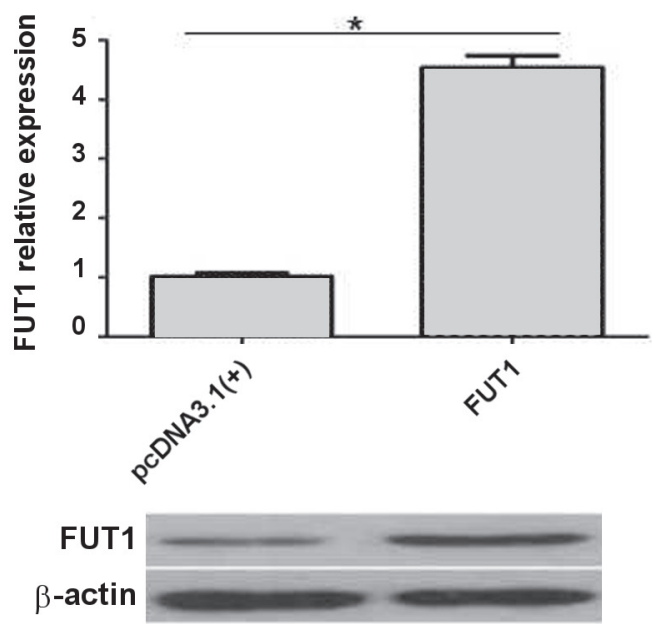

$\mathrm{B}$
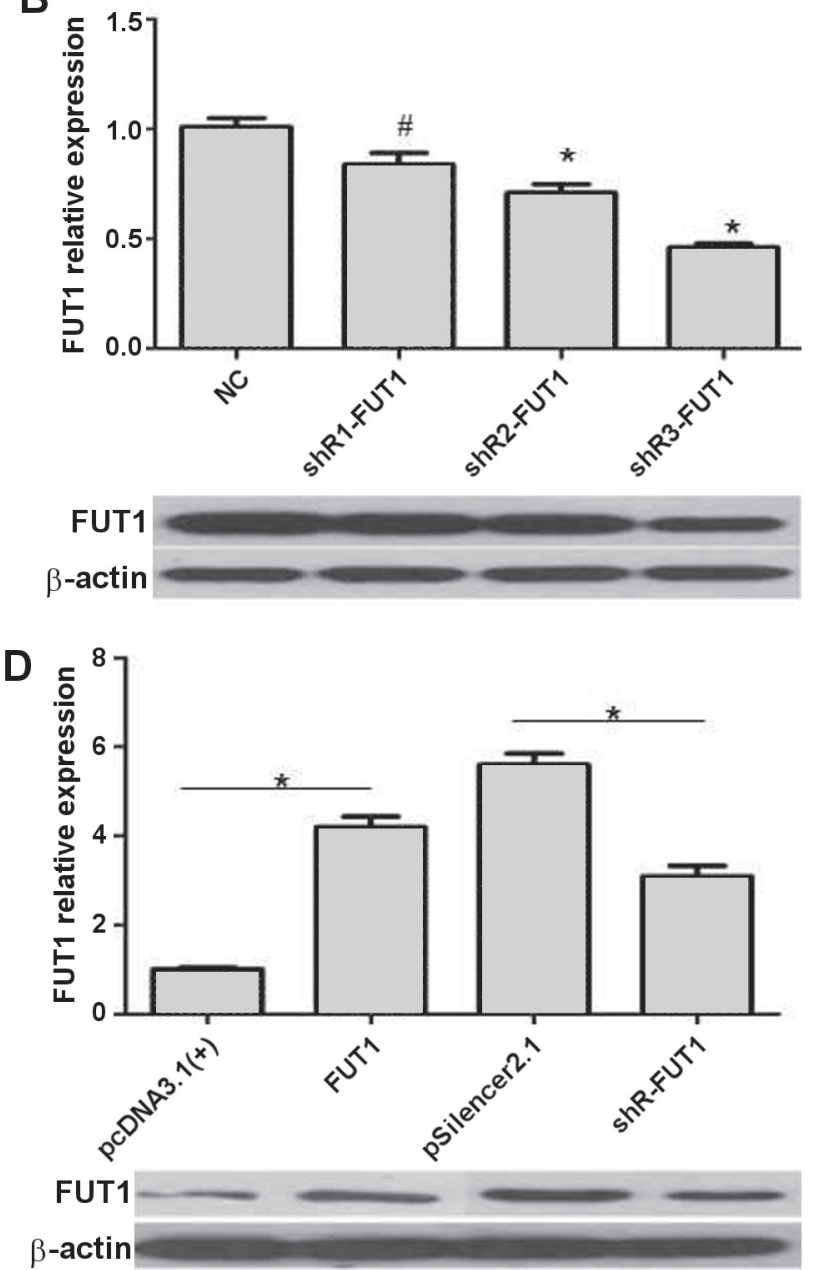

Figure 1. The protein expression of FUT1 in ovarian cancer cell lines, and the establishment of stable cell lines with interference-low and over-expression-high expression of FUT1. A) The western blot results indicate that FUT1 is expressed in the three kinds of epithelial ovarian cancer cell lines, and the expression level is different: ES-2 > SK-OV-3 > OVCAR-3. B) The western blot result of FUT1 protein expression in the shR-FUT1 and control groups. The FUT1 siRNA (shR1, 2 and 3) was transfected into the cells. And the protein level of FUT1 was significantly lower in the shR2/3-FUT1 group than that in the control group $\mathrm{NC}(\mathrm{p}<0.05$, respectively), while the protein level of FUT1 was not significantly lower in the shR2/3-FUT1 group than that in the control group NC ( $>>0.05)$, for the maximum expression of FUT1 cell line ES-2. C) The western blot result of FUT1 protein expression in the FUT1 and control groups. The over-expression plasmid pcDNA3.1-FUT1 was transfected into the cells. And the protein level of FUT1 was significantly higher in the FUT1 group than that in the control group pcDNA3.1 $(+)(p<0.05)$ for the minimum expression of FUT1 cell line OVCAR-3. D) The expression level of FUT1 in the different groups of stable cell line after the treatment with Taxol. Compared with the control group pSilence2.1 transfected with the control shR, the expression level of FUT1 was significantly decreased (p<0.05), and compared with the control group pcDNA3.1(+) transfected with the empty plasmid, the expression level of FUT1 was significantly increased $(P<0.05)$. At least three independent experiments were conducted. Data from three experimental determinations and bars indicate the SD. Data are expressed as the mean \pm standard deviation $\left({ }^{\star} p<0.05\right.$, and \# p>0.05).

The siR-FUT1 combined with Taxol promoted cell apoptosis by regulating the apoptosis-related proteins. Additionally, after the treatment with Taxol, the results of flow cytometry demonstrated that the apoptosis rate was increased or decreased obviously in the shR-FUT1 or FUT1 group compared with the control group $(\mathrm{p}<0.05$, respectively, Figure $2 \mathrm{~B}$ and $\mathrm{C}$ ). However, without the treatment with Taxol, the apoptosis rate was not increased or decreased obviously in the shR-FUT1 or FUT1 group compared with the control group ( $p>0.05$, respectively, Figure $2 B$ and $C$ ).
Taken together, the results demonstrate that siR-FUT1 combined with Taxol could promote cell apoptosis.

Furthermore, to explore the mechanism of the above apoptosis, we tested the expression level of the apoptosis related proteins in the different groups. We tested the level of pro-apoptotic protein caspase-3, and the determination of caspase- 3 enzyme activity test results showed that the caspase- 3 enzyme activity was increased or decreased obviously in the shR-FUT1 (low expression) or FUT1 (high expression) group compared with the control group ( $\mathrm{p}<0.05$, 
respectively, Figure 2D). And the results of western blot indicate that the expression of the anti-apoptotic proteins such as survivin, XIAP, BCL-2 and BCL-xL were significantly decreased or increased in the shR-FUT1 (low expression) or FUT1 (high expression) group compared with the control group $(\mathrm{p}<0.05$, respectively, Figure 3$)$. In summary, our results indicate that FUT1 combined with Taxol could promote cell apoptosis by regulating the apoptosis-related proteins.

Inhibiting effect of siR-FUT1 combined with Taxol against ovarian cancer in vivo. To validate the effect of FUT1 in vivo, we injected ES-2 cells subcutaneously into the right flank of nude mice. The results were as follows: compared to the control group without the Taxol therapy, the average tumor volume in shR-FUT1 group was not decreased significantly ( $p>0.05$, Figure 4$)$. However, after the treatment with Taxol, the in vivo results demonstrated that the tumor volume was decreased obviously in the shR-FUT1 group compared with the control group $(p<0.05$, respectively, Figure 4). Treatment with siR-FUT1 and/or Taxol resulted in no significant difference in the body weight of treated mice, none of the tested mice manifested signs of other adverse effects as specified in the method section, and no toxicity on the blood count or hepatic and renal function was observed (data not shown). These results indicate the anti-tumor effect and safety of siR-FUT1 and/or Taxol in vivo. To sum up, these results suggest that FUT1 could promote development of chemoresistance in ovarian cancer reversely in vivo.
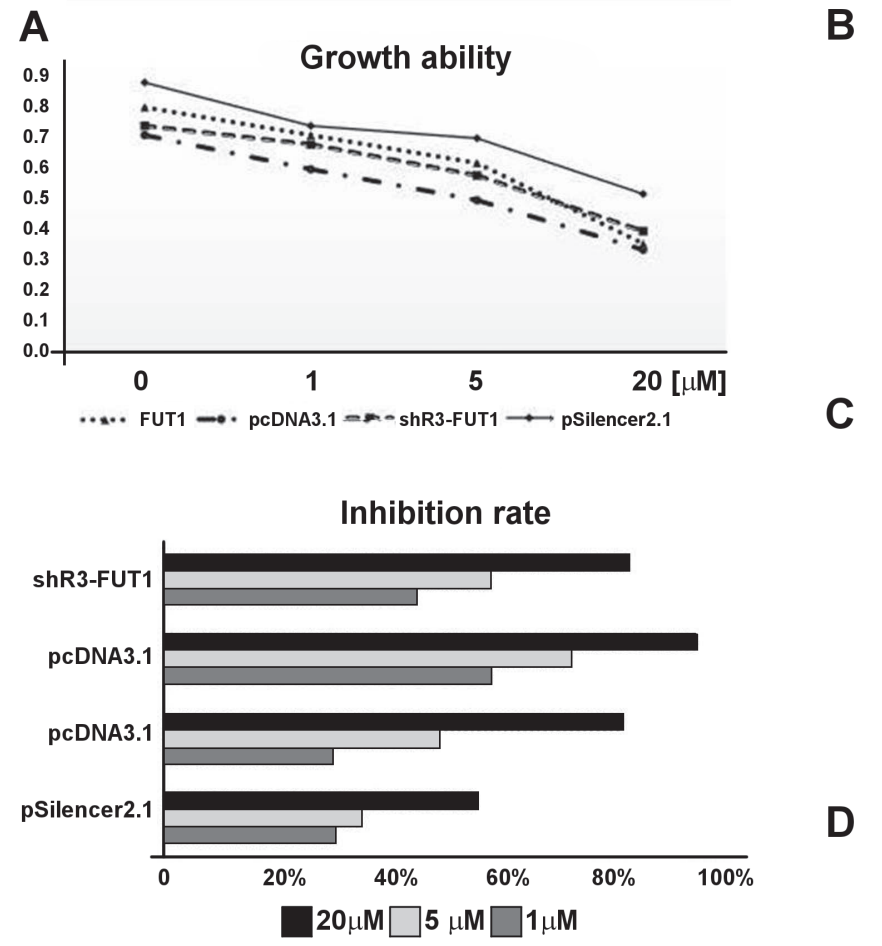

C

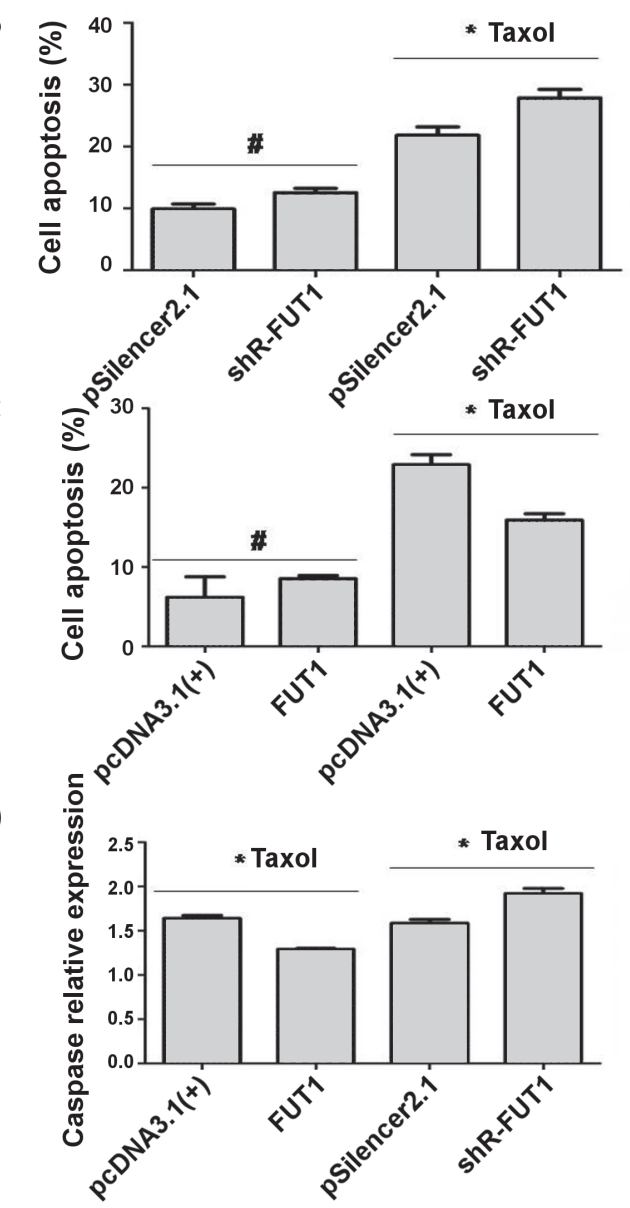

Figure 2. The growth ability, inhibition rate and IC50 level and the results of cell apoptosis rate in the four different stable cell lines. A. The inhibition rate of Taxol was obviously decreased and the IC50 level was significantly increased in the FUT1 group (p $<0.05$, respectively). B, C. The results of flow cytometry demonstrated that the apoptosis rate was increased or decreased obviously in the shR-FUT1 or FUT1 group compared with the control group $(\mathrm{p}<\mathbf{0 . 0 5}$, respectively). However, without the treatment with Taxol, the apoptosis rate was not increased or decreased in the shR-FUT1 or FUT1 group compared with the control group ( $p>0.05$, respectively). $D$. The result of the pro-apoptotic protein caspas- 3 expression in the four different stable cell lines. The determination of caspase- 3 enzyme activity test results showed that the caspase- 3 enzyme activity was increased or decreased obviously in the shR-FUT1 (low expression) or FUT1 (high expression) group compared with the control group (p $<0.05$, respectively). Results are representative of at least three separate experiments. Data are expressed as the mean \pm standard deviation. Data from three experimental determinations and bars indicate the $\mathrm{SD}\left({ }^{*} \mathrm{p}<0.05\right)$. 

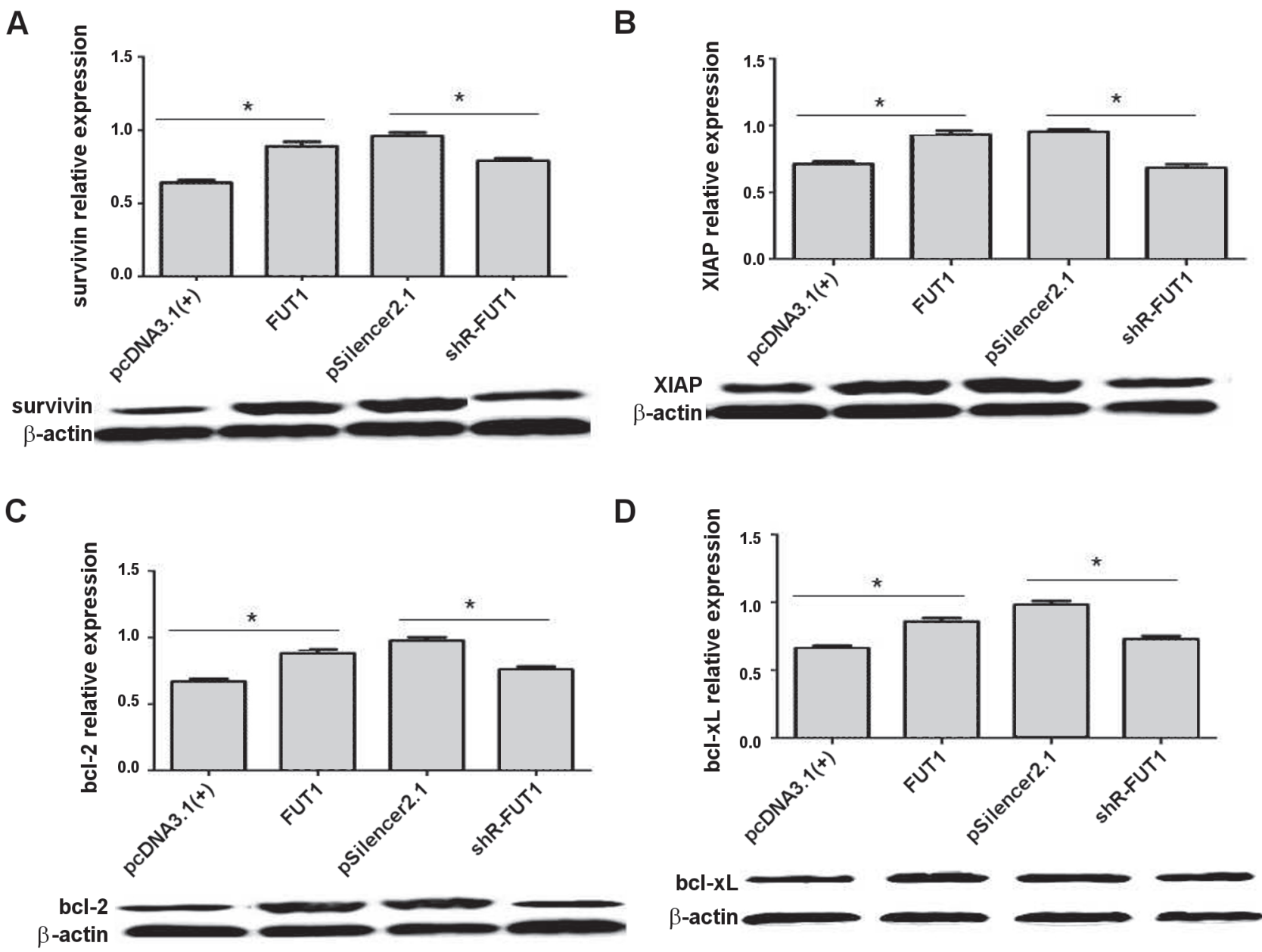

Figure 3. The results of the anti-apoptotic proteins expression in the four different stable cell lines. The results of western blot showed that the expression of the anti-apoptotic proteins such as survivin, XIAP, BCL-2 and BCL-xL was significantly decreased or increased in the shR-FUT1 (low expression) or FUT1 (high expression) group compared with the control group $(\mathrm{p}<0.05$, respectively). Results are representative of at least three separate experiments. Data are expressed as the mean \pm standard deviation. Data from three experimental determinations and bars indicate the SD $\left({ }^{\star} \mathbf{p}<0.05\right)$.

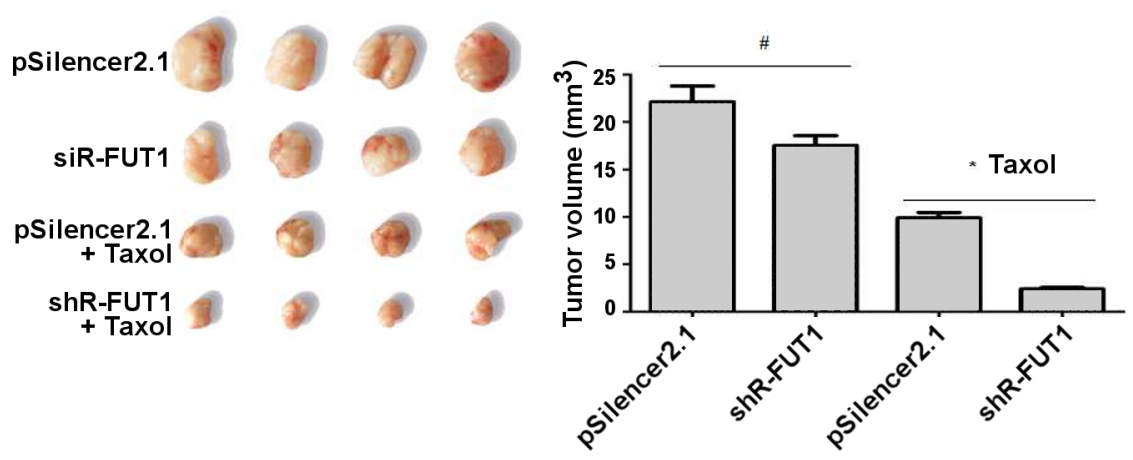

Figure 4. Inhibiting effect of siR-FUT1 combined with Taxol against ovarian cancer in vivo. The tumor volumes in different groups of mice. After 21 days of treatment, the siR-FUT1 combined with Taxol therapy decreased the tumor volumes compared to the control group significantly $(\mathrm{p}<0.05)$. However, compared to the control group, the average tumor volume in siR-FUT1 without Taxol was not decreased significantly ( $p>0.05)$. 
As a whole, our results suggest that FUT1 could promote Taxol resistance and inhibit cell apoptosis by enhancing the expression of anti-apoptotic proteins such as survivin, XIAP, BCL-2, and the BCL, and through the inhibition of caspase-3 enzyme activity.

\section{Discussion}

Taxol is one of the first-line drug of ovarian cancer chemotherapy, but the occurrence of drug resistance has made it challengeable. As a result, exploring the mechanism of drug resistance has become an important direction of current research. Lewis y antigen was reported to be positively correlated with CD147 in resistance to chemotherapeutic drugs in ovarian cancer by using immunohistochemical assays, and the results indicated that CD147 and Lewis y antigen expression were all independent risk factors for chemoresistance in ovarian cancer at the pathological stage [9]. Additionally, a study demonstrated the co-localization of annexin II and Lewis y antigen through the double-labeling immunofluorescence experiments, and that annexin II contained Lewis y antigen [10]. The conclusion was in close correlation between annexin II and Lewis y, both of which were significantly high in ovarian cancer [10]. Besides, another study suggested that over-expression of CD44 and Lewis y were positively correlated in ovarian cancer and both of them were strong risk factors for resistance to chemotherapy in ovarian cancer [11]. Moreover, TGF- $\beta 1$ and human epididymis protein 4 were positively correlated with Lewis y in ovarian cancer [12, 13]. In addition, the over-expression of integrin $\alpha 5 \beta 1$ was brought by the transfection of FUT1, resulting in enhancement of the adhesion and spreading potential of malignant ovarian cells [14-16]. It was also reported that Lewis y could regulate cell cycle related factors via ERK and Akt signaling pathways in ovarian cancer [17]. And Lewis y could regulate the transforming growth factor $\beta$ pathway and up-regulate growth factors in ovarian cancer $[18,19]$. However, in our previous studies, we demonstrated that the over-expression of Lewis y was closely related to the enhancement of the proliferation, drug resistance (including docetaxel-resistance) and metastasis of ovarian cancer [18-20]. Besides, we proved that Lewis y inhibited the apoptosis of ovarian cancer $[18,19]$. Even the anti-Lewis-Y monoclonal antibody was under a phase II trial in ovarian cancer, and consolidation strategies in platinum sensitive disease was currently being tested based on the longer PFS. Yet, the way in which Lewis $y$ antigen participates in ovarian cancer drug resistance still needs further investigation.

It has been well known that FUT1 is one of the key enzymes of Lewis y, and FUT1 plays an important role in the mechanism of Lewis y participating in ovarian cancer. It was also reported that the transfection of FUT1 gene significantly increased the expression of Lewis y antigen [22, 23], promoted the cell proliferation in vitro and tumorigenesis in vivo in ovarian cancer [22]. Consistent with the above results, our results indicate that after the treatment with Taxol, the increased expression of FUT1 promoted chemoresistance and inhibited apoptosis. Moreover, a study indicated that the transfection of FUT1 caused the differential expression of 88 genes in ovarian cancer RMG-1 cells: 60 genes were up-regulated and 28 genes were down-regulated, which were involved in protein binding, nucleotide binding, and cell proliferation, DNA-dependent regulation of transcription, signal transduction, protein amino acid phosphorylation, transcription, and cell adhesion [24]. Another similar study showed the gene expression profile: 215 differentially expressed genes according to the selection criteria (122 up-regulated genes and 93 down-regulated genes). And they found that the differentially expressed genes were involved in BioCarta mammalian target of rapamycin (mTOR) pathway, BioCarta eukaryotic translation initiation factor 4 pathway, and Kyoto Encyclopedia of Genes and Genomes pathways [25]. At last, the genes such as TRIM46, PCF11, BCL6, PTEN, and Lewis Y were further validated [25]. Inspired by the literature, we tried to explain the results of apoptosis from the apoptosis-related proteins such as survivin, XIAP, BCL-2, BCL-xL and caspase-3, which were all mentioned in the literature [23-25]. The results demonstrated that FUT1 could inhibit cell apoptosis by increasing the expression of anti-apoptotic proteins such as survivin, XIAP, BCL-2, and the BCL, and through the inhibition of caspase- 3 enzyme activity, which might help to investigate the molecular mechanism of FUT1 in ovarian cancer.

Chemotherapy is a double-edged sword, which simultaneously leads to the treatment and to the chemoresistance of the tumor. This provides a theoretical basis for the therapy, which needs chemotherapy in combination with the inhibition of FUT1. Even so, future studies are needed to explore more downstream genes and the mechanism of FUT1 during the chemoresistance in ovarian cancer.

In conclusion, our data suggest that FUT1 could promote Taxol resistance and inhibit cell apoptosis by enhancing the expression of anti-apoptotic proteins such as survivin, XIAP, BCL-2, and the BCL, and through the inhibition of caspase-3 enzyme activity. Thus, FUT1 might serve as a therapeutic target for ovarian cancer.

Acknowledgements: This study was supported by the National Natural Science Foundation of China (No. 81402149, 81671434), Scientific and Technological Development Project of Shandong Province (No. 2013WS0114, 2014GSF118048, 20162S0404), Shandong Provincial Natural Science Foundation (No. ZR2013HQ027, 2R2013HM060), Doctoral fund of Shandong Province (No. 2007BS03064).

\section{References}

[1] SIEGEL RL, MILLER KD, JEMAL A. Cancer Statistics, 2017. CA Cancer J Clin 2017; 67: 7-30. https://doi.org/10.3322/ caac. 21387 
[2 NAKAMURA K, SAWADA K, YOSHIMURA A, KINOSE Y, NAKATSUKA E et al. Clinical relevance of circulating cell-free microRNAs in ovarian cancer. Mol Cancer 2016; 15: 48. https://doi.org/10.1186/s12943-016-0536-0

[3] TOUBOUL C, VIDAL F, PASQUIER J, LIS R, RAFII A. Role of mesenchymal cells in the natural history of ovarian cancer: a review. J Transl Med 2014; 12: 271. https://doi. org/10.1186/s12967-014-0271-5

[4] CHEN WT, YANG YJ, ZHANG ZD, AN Q, LI N et al. MiR-1307 promotes ovarian cancer cell chemoresistance by targeting the ING5 expression. J Ovarian Res 2017; 10: 1. https://doi.org/10.1186/s13048-016-0301-4

[5] LIU D, LIU J, WANG C, LIN B, LIU Q et al. The stimulation of IGF-1R expression by Lewis(y) antigen provides a powerful development mechanism of epithelial ovarian carcinoma. Int J Mol Sci 2011; 12: 6781-6795. https://doi.org/10.3390/ ijms 12106781

[6] LIU JJ, LIN B, HAO YY, LI FF, LIU DW et al. Lewis(y) antigen stimulates the growth of ovarian cancer cells via regulation of the epidermal growth factor receptor pathway. Oncol Rep 2010; 23: 833-841.

[7] LIU J, LIN B, HAO Y, QI Y, ZHU L et al. Lewis y antigen promotes the proliferation of ovarian carcinoma-derived RMG-I cells through the PI3K/Akt signaling pathway. J Exp Clin Cancer Res 2009; 28: 154. https://doi.org/10.1186/17569966-28-154

[8] MADJD Z, PARSONS T, WATSON NF, SPENDLOVE I, ELLIS I et al. High expression of Lewis(y)/b antigens is associated with decreased survival in lymph node negative breast carcinomas. Breast Cancer Res 2005; 7, R780-R787. https:// doi.org/10.1186/bcr1305

[9] GAO J, HU Z, LIU J, LIU D, WANG Y et al. Expression of CD147 and Lewis y antigen in ovarian cancer and their relationship to drug resistance. Med Oncol 2014; 31: 920. https:// doi.org/10.1007/s12032-014-0920-9

[10] ZHUANG H, TAN M, LIU J, LI X, GAO J et al. The expression of annexin II and Lewis y antigen in ovarian epithelial tumors and the correlation between them. Tumor Biol 2015; 36: 2343-2349. https://doi.org/10.1007/s13277-014-2841-9

[11] HU Z, GAO J, ZHANG D, LIU Q, YAN L et al. High expression of Lewis y antigen and CD44 is correlated with resistance to chemotherapy in epithelial ovarian cancers. PLoS One 2013; 8: e57250. https://doi.org/10.1371/journal. pone. 0057250

[12] WANG ST, LIU JJ, WANG CZ, LIN B, HAO YY et al. Expression and correlation of Lewis y antigen and TGF- $\beta 1$ in ovarian epithelial carcinoma. Oncol Rep 2012; 27: 10651071. https://doi.org/10.3892/or.2011.1575

[13] ZHUANG H, GAO J, HU Z, LIU J, LIU D et al. Co-expression of Lewis y antigen with human epididymis protein 4 in ovarian epithelial carcinoma. PLoS One 2013; 8: e68994. https://doi.org/10.1371/journal.pone.0068994

[14 HU Z, GAO S, GAO J, HOU R, LIU C et al. Elevated levels of Lewis y and integrin $\alpha 5 \beta 1$ correlate with chemotherapeutic drug resistance in epithelial ovarian carcinoma. Int J Mol Sci 2012; 13: 15588-15600. https://doi.org/10.3390/ ijms 131215588
[15] LI Q, LIU S, LIN B, YAN L, WANG Y et al. Expression and correlation of Lewis y antigen and integrins $\alpha 5$ and $\beta 1$ in ovarian serous and mucinous carcinoma. Int J Gynecol Cancer 2010; 20: 1482-1489. https://doi.org/10.1111/ IGC.0b013e3181ea7ecb

[16] YAN LM, LIN B, ZHU LC, HAO YY, QI Y et al. Enhancement of the adhesive and spreading potentials of ovarian carcinoma RMG-1 cells due to increased expression of integrin alpha5beta1 with the Lewis Y-structure on transfection of the alpha1,2-fucosyltransferase gene. Biochimie 2010; 92: 852-857. https://doi.org/10.1016/j.biochi.2010.02.012

[17] LIU D, LIU J, LIN B, LIU S, HOU R et al. Lewis y regulate cell cycle related factors in ovarian carcinoma cell RMG-I in vitro via ERK and Akt signaling pathways. Int J Mol Sci 2012; 13: 828-839. https://doi.org/10.3390/ijms13010828

[18] LI FF, LIU JJ, LIU DW, LIN B, HAO YY et al. Lewis Y regulates signaling molecules of the transforming growth factor $\beta$ pathway in ovarian carcinoma-derived RMG-I cells. Int J Oncol 2012; 40: 1196-1202. https://doi.org/10.3892/ ijo.2011.1296

[19] LI F, LIN B, HAO Y, LI Y, LIU J et al. Lewis Y promotes growth and adhesion of ovarian carcinoma-derived RMG-I cells by upregulating growth factors. Int J Mol Sci 2010; 11: 3748-3759. https://doi.org/10.3390/ijms11103748

[20] Zhang F, Liu J, Lin B, Liu Q, Zhao Y et al. Increase in docetaxel-resistance of ovarian carcinoma-derived RMG-1 cells with enhanced expression of Lewis Y antigen. Int J Mol Sci 2012; 12: 7323-7334. https://doi.org/10.3390/ijms12117323

[21] SMALETZ O, DIZ MD, DO CARMO CC, SABBAGA J, CUNHA-JUNIOR GF et al. A phase II trial with anti-LewisY monoclonal antibody (hu3S193) for the treatment of platinum resistant/refractory ovarian, fallopian tube and primary peritoneal carcinoma. Gynecol Oncol 2015; 138: 272-277. https://doi.org/10.1016/j.ygyno.2015.05.023

[22] Hao YY, Lin B, Zhao Y, Zhang YH, Li FF et al. [alpha1,2-fucosyltransferase gene transfection influences on BIOLOGICAL behavior of ovarian carcinoma-derived RMG-I cells]. Fen Zi Xi Bao Sheng Wu Xue Bao 2008; 41: 435-442.

[23] LIN B, HAO YY, WANG DD, ZHU LC, ZHANG SL et al. [Transfection of alpha1,2-fucosyltransferase gene increases the antigenic expression of Lewis y in ovarian cancer cell line RMG-I]. Zhongguo Yi Xue Ke Xue Yuan Xue Bao 2008; 30: 284-289.

[24] ZHU LC, LIN B, HAO YY, LI FF, DIAO B et al. [Impact of alpha1,2-fucosyl transferase gene transfection on cancerrelated gene expression profile of human ovarian cancer cell line RMG-1]. Ai Zheng 2008; 27: 934-941.

[25] GAO S, ZHU L, FENG H, HU Z, JIN S et al. Gene expression profile analysis in response to a1,2-fucosyl transferase (FUT1) gene transfection in epithelial ovarian carcinoma cells. Tumour Biol 2016; 37: 12251-12262. https://doi. org/10.1007/s13277-016-5080-4 